\title{
Clinical Characteristics and Outcomes of Community-Acquired versus Hospital-Acquired Acute Kidney Injury: A Meta-Analysis
}

\author{
Linxi Huang $^{a}$ Cheng Xue $^{a}$ Jianke Kuai $^{b}$ Mengna Ruan ${ }^{a}$ Bo Yang ${ }^{a}$ \\ Xujiao Chen $^{a}$ Yu Zhang ${ }^{c}$ Yixin Qian $^{a}$ Jun Wu ${ }^{a}$ Xuezhi Zhao ${ }^{a}$ \\ Changlin $\mathrm{Mei}^{\mathrm{a}}$ Jing $\mathrm{Xu}^{\mathrm{a}}$ Zhiguo Mao ${ }^{\mathrm{a}}$ \\ aDivision of Nephrology, Kidney Institute of CPLA, Changzheng Hospital, Second Military \\ Medical University, Shanghai, China; ${ }^{b}$ Third Hospital of Xi'an, Department of Anesthesiology, \\ Xi'an, China; 'Medical team of 32120 troop of PLA, Dalian, China
}

\section{Keywords}

Acute kidney injury · Community-acquired · Hospital-acquired $\cdot$ Clinical characteristics

\begin{abstract}
Background: The different clinical characteristics of community-acquired acute kidney injury (CA-AKI) versus hospital-acquired AKI (HA-AKI) have remained inconclusive, and thus, a metaanalysis was conducted to summarize and quantify the clinical significance distinguishing the 2 types of AKI. Methods: We identified observational studies reporting the clinical characteristics and prognosis of HA-AKI and CA-AKI. ORs and mean differences (MDs) were extracted for each outcome and the results aggregated. The primary outcome was defined as the mortality rate; renal recovery, oliguria incidence, dialysis, intensive care unit (ICU) requirement, and length of hospital stay were secondary outcomes. Results: Fifteen eligible studies involving 46,157 patients (22,791 CA-AKI patients and 23,366 HA-AKI patients) were included. Mortality was significantly lower in CA-AKI than in HA-AKI patients, with an OR of $0.43(95 \% \mathrm{Cl}$ 0.35-0.53). The incidence of oliguria and need for ICU were also lower in CA-AKI patients (OR $0.58,95 \% \mathrm{Cl} 0.38-0.88$; OR $0.24,95 \% \mathrm{Cl} 0.14-0.40$, respectively). CA-AKI patients had a shorter hospital stay (MD $-9.42,95 \% \mathrm{Cl}-13.73$ to -5.12 ). The renal recovery rate and dialysis need between CA- and HA-AKI were similar (OR 1.27, 95\% Cl 0.53-3.02; OR 1.05, 95\% Cl 0.82-1.34, respectively). Conclusions: CA-AKI showed better clinical manifestations with a lower incidence of oliguria, reduced risk of ICU treatment, and shorter hospital stay. Mortality associated with CA-AKI was lower compared with HA-AKI, indicating a better prognosis. The rate of renal recovery and need for dialysis showed no significant difference between the 2 groups.

L.H., C.X., J.K., and M.R. contribute equally to the research. 


\section{Kidney \\ Blood Pressure \\ Research}

\begin{tabular}{l|l}
\hline Kidney Blood Press Res 2019;44:879-896 \\
\hline DOI: 10.1159/000502546 & $\begin{array}{l}\text { @ 2019 The Author(s). Published by S. Karger AG, Basel } \\
\text { www.karger.com/kbr }\end{array}$ \\
\hline
\end{tabular}

Huang et al.: Comparison of CA-AKI and HA-AKI

\section{Introduction}

Acute kidney injury (AKI) is a syndrome characterized by a rapid loss of kidney function and clinically diagnosed by the accumulation of nitrogenous metabolites (urea and creatinine), decreased urine output, or both [1-6]. It was first recognized during the air raids over London decades ago and is still a major contributor to poor patient outcomes, currently occurring in approximately 13.3 million people per year $[7,8]$.

Hospital-acquired AKI (HA-AKI) has long been a research focus, especially in the intensive care unit (ICU) [9]. In 1991, Kaufman etal. [10] reported patients who had presented acutely increased serum urea and creatinine levels at admission to hospital and initiate the diagnosis of community-acquired AKI (CA-AKI). With improving diagnostic and therapeutic modalities and the rapidly aging population worldwide, the incidence of AKI has been reported to be rising, and CA-AKI seems to dominate [11]. Nevertheless, confirmed epidemiological data for AKI are scarce, and the available data vary among regions and countries [12]. Loo and Zainal [13] reported 2 AKI patients per 1,000 admissions based on data from a single hospital in Malaysia. Studies conducted in southern Saudi Arabia and North India reported an AKI incidence upon hospitalization of approximately $0.6 \%[14,15]$. A systematic review of large cohort studies provided an AKI world incidence estimate of 1 in 5 adults and 1 in 3 children during hospital care [16]. In Taiwan, the incidences of CA-AKI and HA-AKI were separately calculated as 17.25 and 8.14 per 1,000 admissions, and the annual rate of CA-AKI was increasing, while that of HA-AKI was maintained [17]. A large cross-sectional study conducted in China indicated that CA-AKI patients made up $54.4 \%$ of all hospitalized AKI patients [18].

The differences in clinical characteristics between CA-AKI and HA-AKI patients are controversial. Similar long-term sequelae of CA-AKI and HA-AKI patients, such as renal disease progression and mortality, have been reported, while higher mortality has been observed for CA-AKI patients $[19,20]$. Xu et al. [5] reported that the dialysis requirement rate for CA-AKI was $4.5 \%$, which was significantly higher than that for HA-AKI of 3.5\% $(p<0.001)$. Hsu et al. [17] reported the opposite result, with a dialysis rate of $2.06 \%$ for HA-AKI compared with $1.45 \%$ for CA-AKI $(p<0.001)$. Thus, it has become necessary to conduct a study that compares the characteristics of CA- and HA-AKI to show the complete landscape of their difference. Moreover, the application of different criteria in published studies for the diagnosis of AKI complicate data comparisons [16, 21,22]. Thus, here we attempted to surmount the above-mentioned possible obstacles to summarize and provide comprehensive and convincing evidence.

The present meta-analysis aims to comprehensively quantify and classify the differences in CA- and HA-AKI patients both in demography, the degree of need for clinical care, and prognosis, especially in mortality and renal recovery, which will be helpful for evidence-based clinical decision-making.

\section{Methods}

We conducted a comprehensive search of the medical literature using PubMed, EMBASE, Cochrane, and Web of Science from their inceptions to January 2019. The PubMed search terms (both as medical subject headings and free text terms) were as follows: (CA-AKI) and (HA-AKI). We additionally searched the reference lists of the original reports, reviews, guidelines, and meta-analyses retrieved through the electronic searches. Only publications in English were retrieved. 


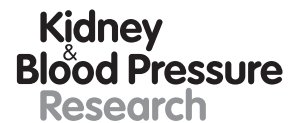

Kidney
Blood Pressure
Research

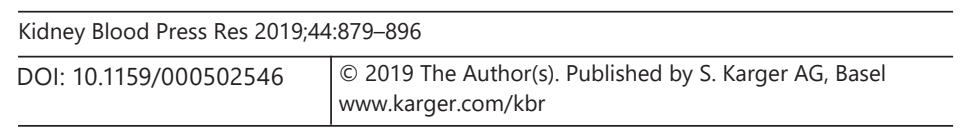

Huang et al.: Comparison of CA-AKI and HA-AKI

We selected the studies in 2 steps. First, 2 review authors (L.H. and J.X.) independently screened the titles and abstracts. Second, the full texts of potentially eligible studies were retrieved and assessed independently by the same 2 authors. The pre-specified eligibility criteria were as follows: (1) observational studies, either retrospective or prospective studies; (2) mentioned both CA-AKI and HA-AKI patients; (3) made comparisons between CA and HA-AKI patients; (4) mentioned clinical characteristics, particularly mortality. Studies using other observational methods were eligible as long as there were comparable data related to both CA and HA-AKI patients. We excluded studies with the following properties: (1) case report; (2) studies focusing on pediatric patients; (3) mono-drug-induced AKI. We identified 2 articles that used the same patient samples, and we included the 1 with the most relevant information $[23,24]$. Any disagreement between review authors was resolved by consensus, adjusted with the support of a third review author (C.X.).

\section{Data Extraction}

Two authors (L.H. and M.R.) extracted all data independently into a predesigned data collection form (Microsoft Office Excel 2010; Microsoft Corp, Redmond, WA, USA). A third review author (Z.M.) checked all the extracted data. The following data were extracted for each study: study design; geographical setting of the study; follow-up year; sample size; patient characteristics (age; proportions of male and female patients; and AKI stages); detailed relevant medical management; duration of hospital stay; and number of patients along with detailed information about the clinical characteristics and prognosis. We defined our primary outcome as the mortality of CA and HA-AKI patients. As renal recovery compromises the prognosis of AKI patients, it was combined as a secondary outcome; oliguria occurrence, dialysis need, ICU specialized care, and length of hospital stay were also analyzed as secondary outcomes. We attempted to contact the original investigators to obtain further information if necessary.

\section{Assessment of the Risk of Bias}

Assessment of the risk of bias was performed independently by 2 review authors (J.K. and C.X.), with disagreements resolved by discussion. Risks of bias ratings for each study were evaluated according to the quality domains in the Cochrane risk of bias tool Nosgen-ranking. Risk of bias for each domain was rated as high (seriously weakens confidence in the results), unclear, or low (unlikely to seriously alter the results).

\section{Data Synthesis and Statistical Analysis}

For retrospective studies, heterogeneity among studies was evaluated by calculating the $I^{2}$ statistic and chi-square test (assessing the $p$ value). If the $p$ value was $<0.10$ and $I^{2}$ exceeded $50 \%$, we considered the heterogeneity substantial. We used the random effects model to combine data with substantial heterogeneity among studies. We summarized dichotomous data for patient properties as the OR. Continuous data were pooled as the mean difference (MD) with 95\% CIs. We conducted pre-specified subgroup analyses according to the baseline properties and prognosis of the subjects. Sensitivity analyses were carried out by excluding outliers and studies with a small sample size to test the stability of our results.

Review Manager version 5.3 (Copenhagen: The Nordic Cochrane Centre, The Cochrane Collaboration, 2012) was used to generate forest and funnel plots. The funnel plots were assessed for evidence of asymmetry and other small study effects. We also assessed the publication bias via Egger's test. We followed the preferred reporting items for systematic reviews and meta-analyses (PRISMA) guidelines in reporting our findings. 


\section{Kidney \\ Blood Pressure \\ Research}

\begin{tabular}{l|l}
\hline Kidney Blood Press Res 2019;44:879-896 \\
\hline DOI: 10.1159/000502546 & $\begin{array}{l}\text { @ 2019 The Author(s). Published by S. Karger AG, Basel } \\
\text { www.karger.com/kbr }\end{array}$ \\
\hline
\end{tabular}

Huang et al.: Comparison of CA-AKI and HA-AKI

\section{Results}

The search strategy initially identified 137 records, of which 48 were potentially relevant to our meta-analysis, for which the full texts were retrieved for further evaluation (Fig. 1). Of these, 32 were excluded according to the inclusion/exclusion criteria, leaving a total of 16 eligible articles. Two articles described results from the same research with different focuses, and thus we adopted the more relevant of the 2 . Finally, all included studies comprised 46,157 patients for the quantitative synthesis and meta-analysis.

\section{Study Characteristics}

Table 1 shows the characteristics of the included studies. Subjects in 15 studies were all AKI patients. Seven studies [16-18, 20-23] were based on data from mono-centers, while the rest were based on multicenters. Among all 46,157 AKI patients, 22,791 were confirmed as community-acquired. The definitions of CA and HA-AKI were clearly described in all the included studies. Regarding the AKI definitions, Loo and Zainal [13], Obialo et al. [25], Al-Homrany [14], and Wang et al. [24] required serum creatinine (SCr) $>2 \mathrm{mg} / \mathrm{dL}$. The KDIGO criteria [21, 26] were applied in 6 studies [5, 12, 18, 27-29], RIFLE [30] was applied in 4 studies [15, 18, 19, 23], and Sesso et al. [31] defined AKI as a SCr level $>1.4 \mathrm{mg} / \mathrm{dL}(124 \mathrm{~mol} / \mathrm{L})$ for men or $>1.3 \mathrm{mg} / \mathrm{dL}(115 \mathrm{~mol} / \mathrm{L})$ for women. Mortality was compared in 14 studies [5, 13, 14, 17-20, 24, 25, 27-29, 31, 32], among which 11 studies compared in-hospital mortality; Wonnacott et al. [28] compared in-hospital and 14-month mortality; Sawhney et al. [27] provided 30-day, 1-year, and 5-year mortality; and Holmes et al. [29] compared 90-day mortality. To reduce bias due to data extraction, we collected the 30-day mortality data from Sawhney et al. [27] and the overall mortality from Wonnacott et al. [28]. Nine studies [12, 14, $17,20,24,27-29,32]$ compared renal recovery, but the criteria differed among all the included studies. Nevertheless, a decline in SCr to almost the normal level was at least required for renal recovery. Sawhney et al. [27] and Mehta et al. [12] classified partial and full recovery. Thus, we took the sum of the 2 kinds of recovery data to reduce bias in the prognosis analysis in our study. Three studies [24, 25, 31] compared oliguria, and 11 studies $[5,13,14,17,20$, $24,25,28,29,31,32]$ compared dialysis rate. Eight studies $[5,13,20,24,25,27,28,32]$ calculated the ICU hospitalization period, and 9 studies [5, 13, 17, 20, 24, 25, 28, 29, 32] compared the length of hospital stay.

\section{Evidence from the Included Studies}

Mortality

Fourteen studies involving 42,204 patients were pooled. As shown in Figure 2, mortality was significantly lower in the CA-AKI than in HA-AKI patients (OR 0.43, 95\% CI 0.35-0.53, $\left.p<0.00001, I^{2}=90 \%\right)$. Exclusion of studies with a small sample size $(n=100)$ did not change the effect size nor the degree of heterogeneity (OR 0.43, 95\% CI $0.35-0.54, I^{2}=91 \%, p<$ $0.00001)$. Subgroup analyses of different AKI definitions provided consistent results with the overall analysis (SCr $>2 \mathrm{mg} / \mathrm{dL}$ : OR $0.26,95 \% \mathrm{CI} 0.18-0.37, p<0.00001, I^{2}=0 \%$; KDIGO: OR $0.48,95 \%$ CI $0.36-0.63, p<0.00001, I^{2}=92 \%$; RIFLE: OR 0.51, 95\% CI 0.26-0.99, $p=0.05$, $I^{2}=94 \%$; Sesso et al. [31]: OR 0.36, 95\% CI 0.23-0.56, $p<0.00001$ ). Schissler et al. [32] reported detailed data for mortality in different RIFLE stages, and we then conducted a subgroup analysis. The results showed that in stages of "Risk," "Injury," and "Failure," respectively, CA-AKI patients had a lower mortality than HA-AKI patients (RIFLE R: OR 0.16, 95\% CI 0.06-0.46, $p=0.0006$; RIFLE I: OR 0.29, 95\% CI 0.12-0.69, $p=0.005$; RIFLE F: OR 0.21, $95 \%$ CI $0.07-0.61, p=0.004)$. We did not identify publication bias based on Egger's test ( $p=$ $0.868)$. 


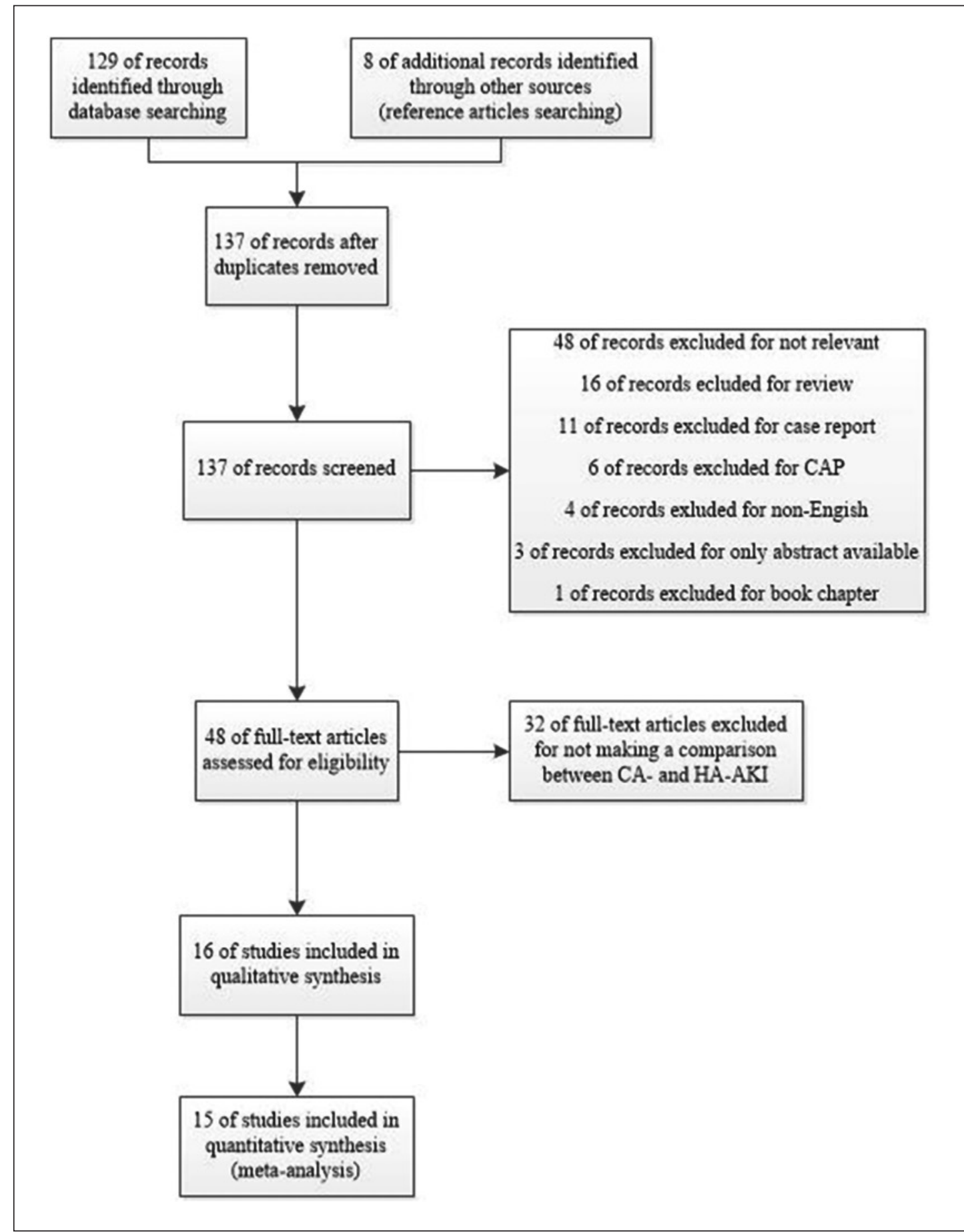

Fig. 1. Flow chart of the included studies. HA-AKI, hospital-acquired acute kidney injury; CA-AKI, community-acquired acute kidney injury. 
Kidney

Blood Pressure

Research
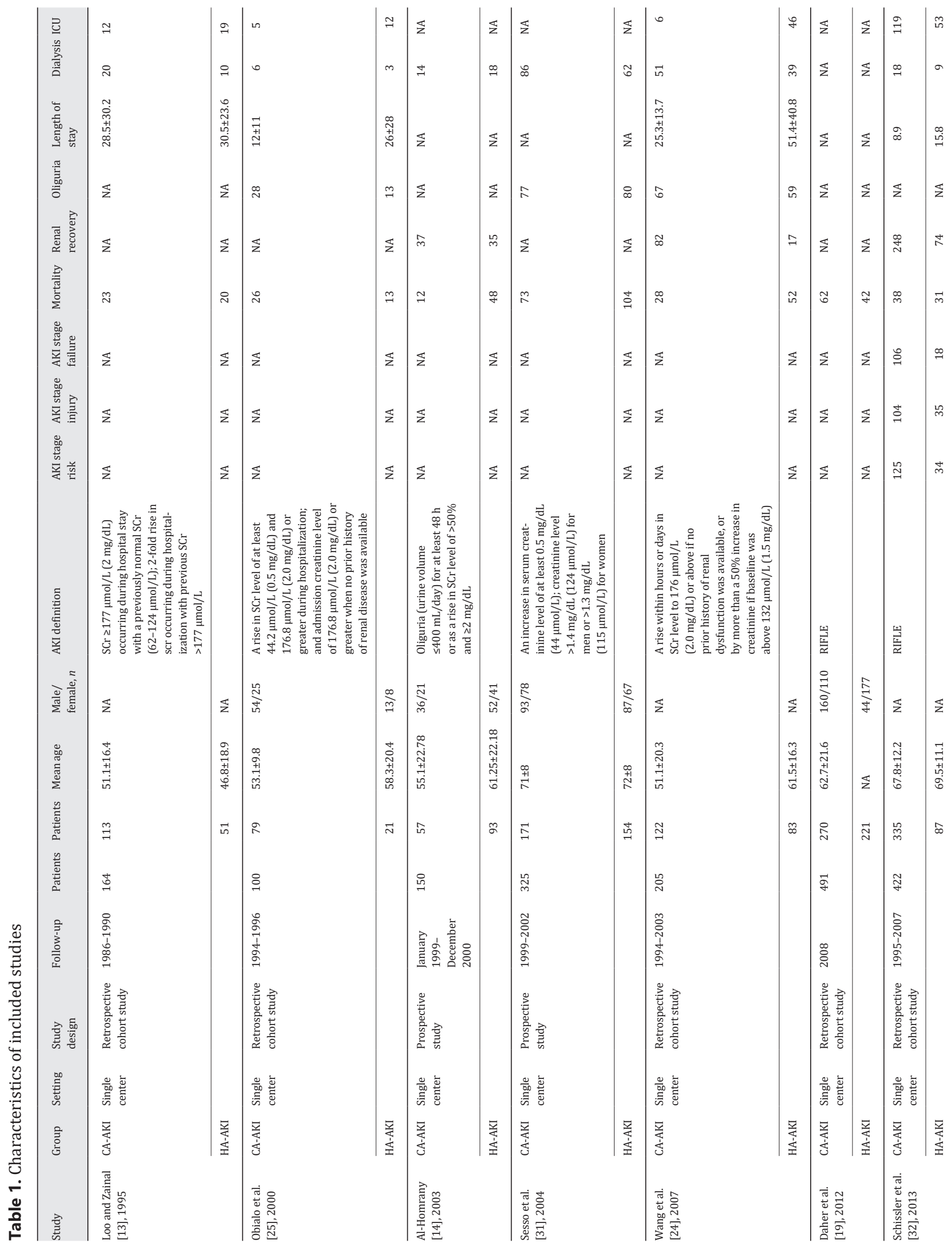

$\mathbb{z}$

\begin{tabular}{ll|l|l|l}
$\mathbb{z}$ & $\mathbb{z}$ & $\mathbb{z}$ & $\stackrel{\text { I }}{\exists}$ & $\mathbb{m}$
\end{tabular}

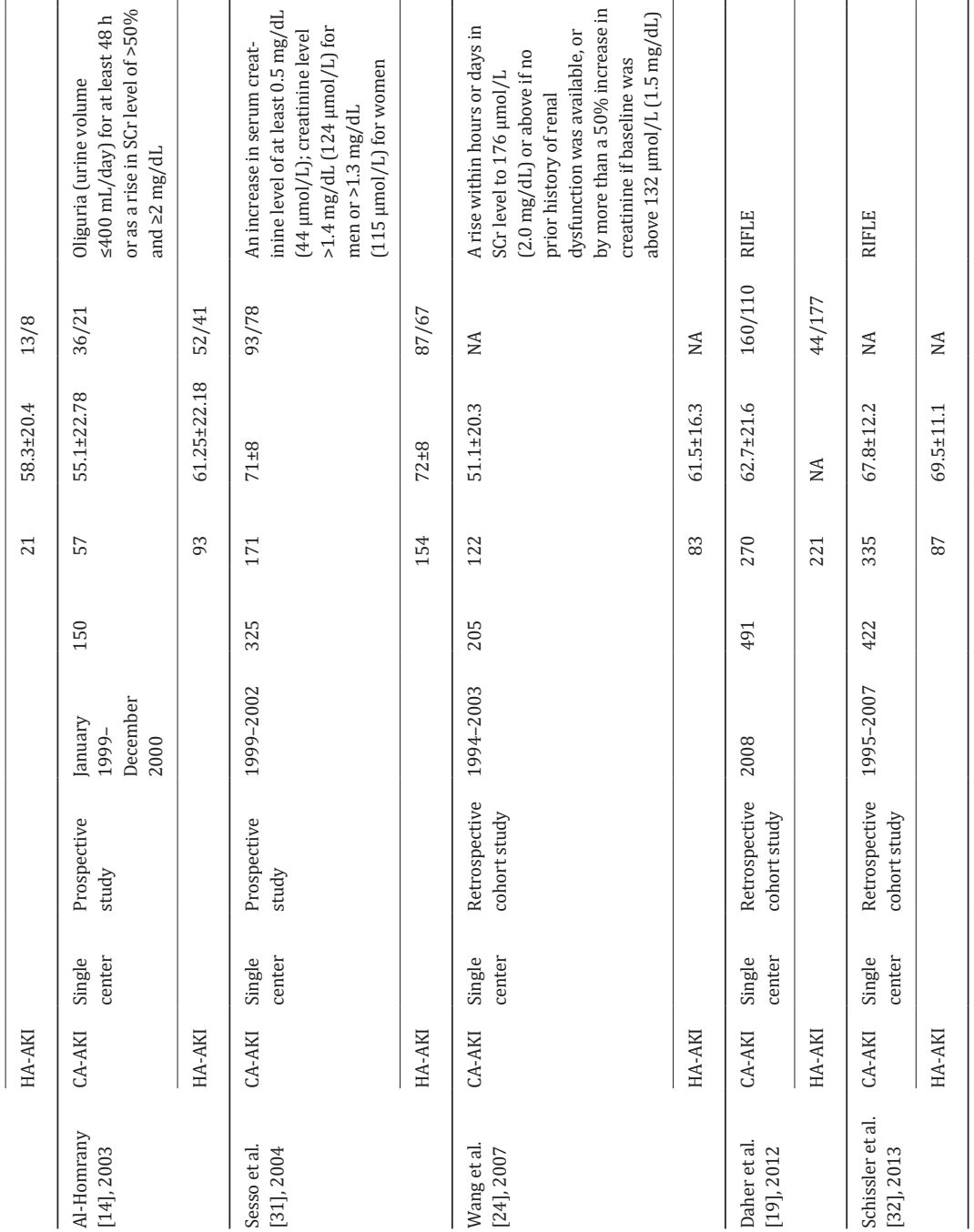




\begin{tabular}{|c|c|c|c|c|c|c|c|c|c|c|c|c|c|c|c|c|c|}
\hline$\underset{n}{\underline{Z}}$ & $\stackrel{2}{\exists}$ & N & $\tilde{m}$ & 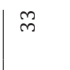 & $\overrightarrow{\vec{m}}$ & ్ָ & $\frac{\pi}{z}$ & $\frac{\pi}{z}$ & $\stackrel{\infty}{\underset{\sim}{+}}$ & $\stackrel{\infty}{\stackrel{\infty}{\infty}}$ & $\overleftrightarrow{z}$ & $\mathbb{z}$ & $\mathbb{z}$ & $\overleftarrow{z}$ & $\frac{\pi}{z}$ & $\frac{\pi}{z}$ & \\
\hline 产 & $\stackrel{\mathrm{N}}{ }$ & $\infty$ & $\stackrel{\sim}{\sim}$ & ${ }^{\circ}$ & 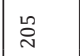 & 总 & $\bar{z}$ & $\frac{\pi}{z}$ & $\frac{\pi}{z}$ & $\frac{\pi}{z}$ & $\frac{\pi}{z}$ & $\frac{\pi}{z}$ & $\vec{\sigma}$ & 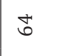 & 용 & ¿ & \\
\hline 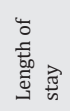 & $\stackrel{\sharp}{ت}$ & స్తి & $\begin{array}{l}\frac{0}{0} \\
\overline{1} \\
0 \\
n\end{array}$ & 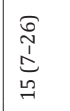 & 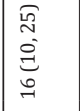 & 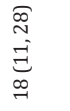 & $\mathbb{z}$ & $\frac{\pi}{z}$ & $\overleftrightarrow{z}$ & $\mathbb{z}$ & $\mathbb{z}$ & $\frac{\pi}{z}$ & 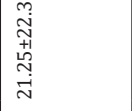 & 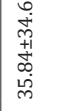 & 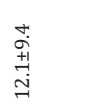 & $\stackrel{\infty}{\sim}$ & \\
\hline 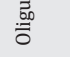 & $\frac{\pi}{z}$ & $\mathbb{z}$ & $\tilde{z}$ & $\tilde{z}$ & $\overleftrightarrow{z}$ & $\tilde{z}$ & $\tilde{z}$ & $\tilde{z}$ & $\overleftrightarrow{z}$ & $\pi$ & $\pi$ & $\frac{\pi}{z}$ & $\mathbb{z}$ & $\frac{\pi}{z}$ & $\overleftarrow{z}$ & $\mathbb{z}$ & \\
\hline 焉 & $\stackrel{\infty}{\sim}$ & $\stackrel{2}{n}$ & 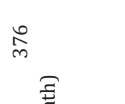 & 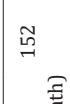 & ż & $\tilde{z}$ & $\tilde{z}$ & $\mathbb{z}$ & \% & 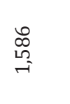 & 总 & 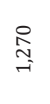 & 雚 & $\stackrel{\mathscr{2}}{q}$ & $\stackrel{n}{\wedge}$ & $\stackrel{q}{\xi}$ & \\
\hline 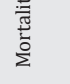 & $\stackrel{\text { I }}{ }$ & F & 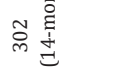 & 号 总 & $\underset{\sim}{*}$ & $\underset{\mathrm{g}}{\mathrm{g}}$ & 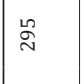 & $\tilde{\sigma}$ & $\stackrel{\sim}{\tilde{i}}$ & ભ̊ & $\mathbb{z}$ & $\frac{\pi}{z}$ & 畺 & $\vec{G}_{-i}^{\vec{j}}$ & 葉 & $\stackrel{m}{m}$ & \\
\hline 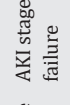 & 导 & \& & $\stackrel{\llcorner\infty}{\sim}$ & '8 & 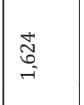 & 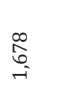 & $\tilde{z}$ & $\frac{\pi}{z}$ & $\underset{-}{\stackrel{N}{*}}$ & $\cong$ & $\frac{\pi}{z}$ & $\frac{\pi}{z}$ & $\begin{array}{l}\stackrel{0}{0} \\
\infty \\
\infty \\
\infty\end{array}$ & 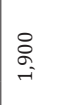 & $\vec{D}$ & $N$ & 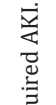 \\
\hline 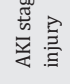 & $\stackrel{\triangle}{\sim}$ & 员 & $\stackrel{\text { ते }}{\text {. }}$ & $\tilde{\alpha}$ & 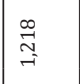 & $\underset{-\underset{T}{N}}{\stackrel{N}{*}}$ & $\tilde{z}$ & $\mathbb{z}$ & 岺 & $\stackrel{\circ}{\vec{m}}$ & $\widetilde{z}$ & $\tilde{z}$ & 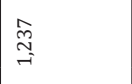 & 品 & $\stackrel{\infty}{\sim}$ & 号 & 䆓 \\
\hline 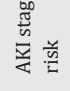 & $\stackrel{\Xi}{\sim}$ & in & స్ & $\stackrel{M}{=}$ & 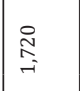 & $\begin{array}{c}\text { M. } \\
\text { S. } \\
\text { o. }\end{array}$ & $\tilde{z}$ & $\mathbb{z}$ & $\underset{\sim}{\stackrel{\tilde{m}}{\sim}}$ & 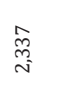 & $\tilde{z}$ & $\mathbb{z}$ & $\underset{ت}{\stackrel{ \pm}{二}}$ & 占 & 芝 & ?్తి & $\begin{array}{l}\text { ह } \\
\vec{\Xi} \\
\vec{\Xi}\end{array}$ \\
\hline $\begin{array}{l}\frac{\bar{g}}{\bar{v}} \\
\frac{\bar{v}}{4}\end{array}$ & 崫 & & 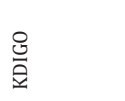 & & 号 & & 蒙 & & 导 & & $\begin{array}{l}\stackrel{\circ}{0} \\
\stackrel{0}{10}\end{array}$ & & 层 & & 을 & & 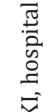 \\
\hline 章 & 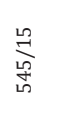 & $\stackrel{m}{\stackrel{m}{\mathrm{~h}^{n}}}$ & 辛 & $\underset{-1}{\stackrel{n}{E}}$ & 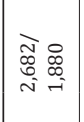 & 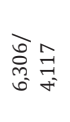 & $\mathbb{z}$ & $\mathbb{z}$ & 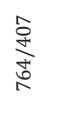 & 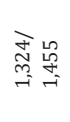 & $\mathbb{z}$ & $\tilde{z}$ & 恕 & 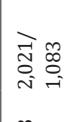 & 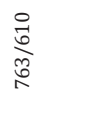 & 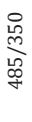 & 竞 \\
\hline 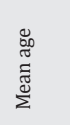 & 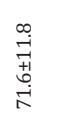 & 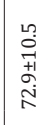 & 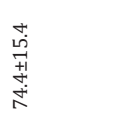 & 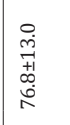 & 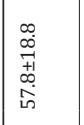 & 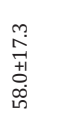 & $\overleftrightarrow{z}$ & $\tilde{z}$ & $\overleftrightarrow{z}$ & $\stackrel{R}{i}$ & $\overleftarrow{z}$ & 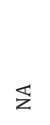 & 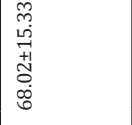 & $\mid \begin{array}{l}0 \\
0 \\
0 \\
01 \\
0 \\
0 \\
0 \\
0\end{array}$ & 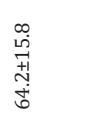 & 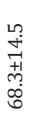 & 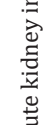 \\
\hline 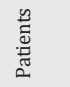 & : & 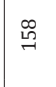 & 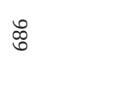 & 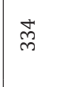 & 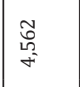 & 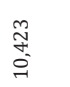 & 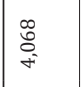 & 产 & $\underset{ت}{\stackrel{5}{E}}$ & 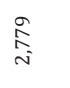 & 僁 & : & 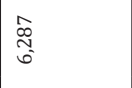 & $\underset{m}{\stackrel{+}{m}}$ & 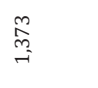 & $\mathscr{\infty}_{\infty}^{L}$ & $\overrightarrow{\tilde{z}}$ \\
\hline 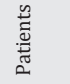 & $\stackrel{\infty}{\sim}$ & & 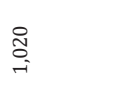 & & 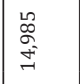 & & 足 & & 品 & & & & & & & & 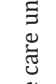 \\
\hline $\begin{array}{l}\text { 言 } \\
\text { 言 } \\
\text { 案 }\end{array}$ & 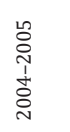 & & $\begin{array}{l}\text { ज̃ } \\
\text { İ } \\
\text { ते }\end{array}$ & & 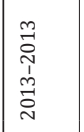 & & $\stackrel{m}{\vec{N}}$ & & & & 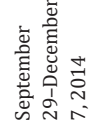 & & 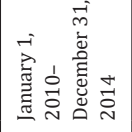 & & 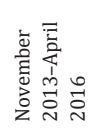 & & 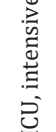 \\
\hline 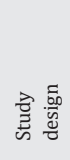 & 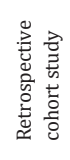 & & 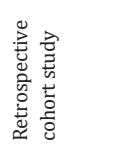 & & 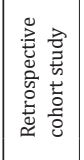 & & 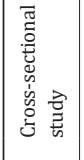 & & 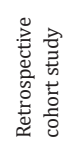 & & 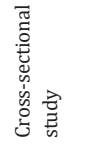 & & 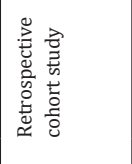 & & 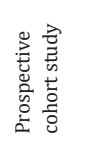 & & 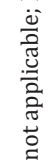 \\
\hline 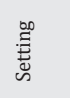 & 竞离 & & 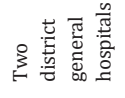 & & 訔耊 & & 言密 & & 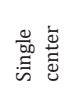 & & 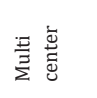 & & 害离 & & 害总 & & 范 \\
\hline 言 & 焉 & 妾 & & 妾 & 弯 & 妾 & 妾 & 妾 & 妾 & 妾 & 妾 & 妾 & 妾 & 妾 & & $\frac{\bar{y}}{\frac{1}{x}}$ & 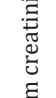 \\
\hline$\frac{z}{z}$ & 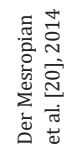 & & 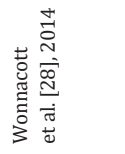 & & 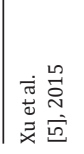 & & 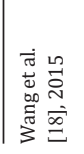 & & 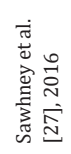 & & 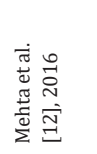 & & 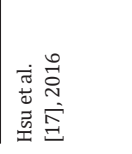 & & 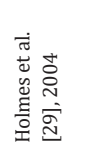 & & 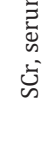 \\
\hline
\end{tabular}




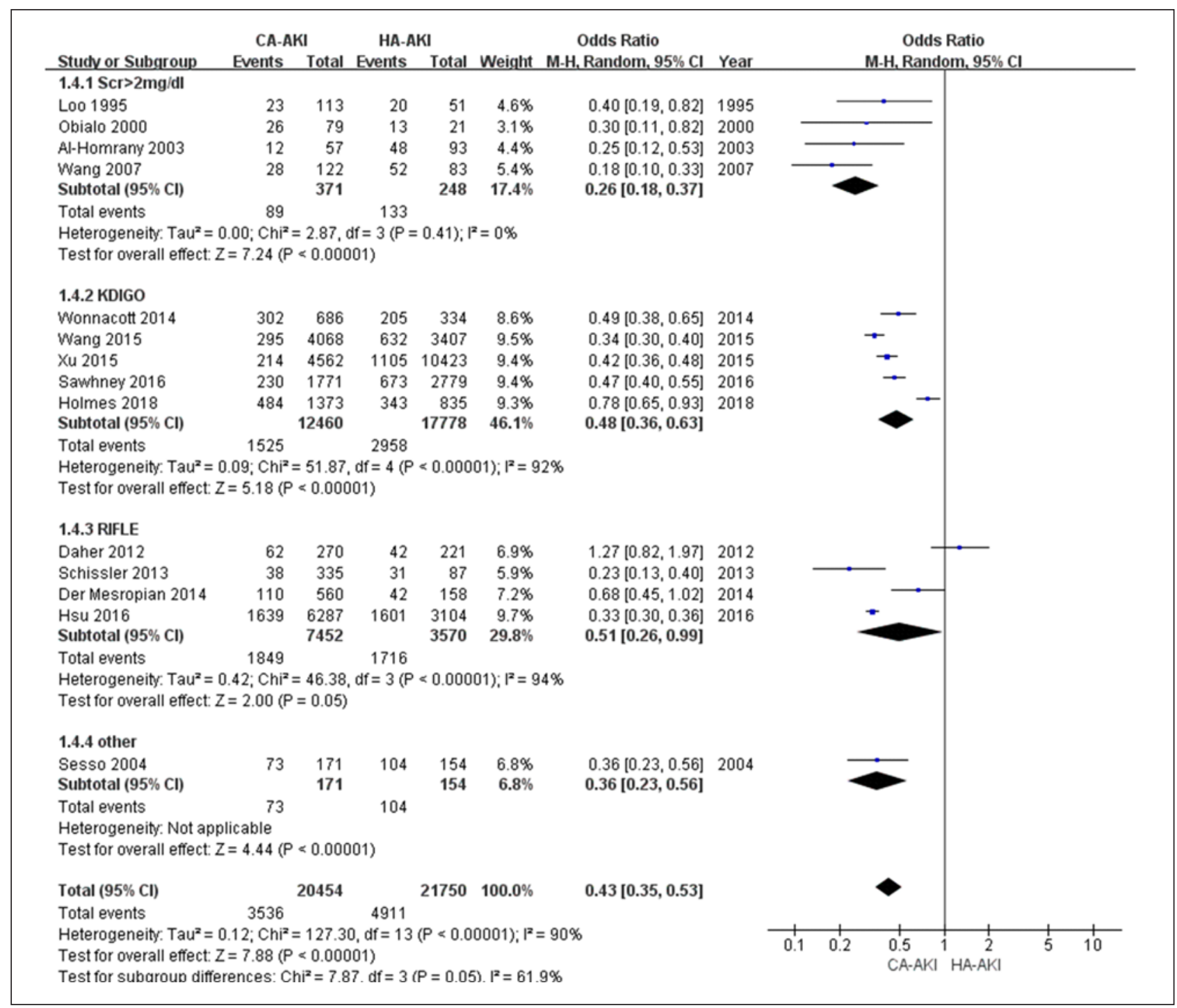

Fig. 2. Forest plot of mortality between CA- and HA-AKI patients. HA-AKI, hospital-acquired acute kidney injury; CA-AKI, community-acquired acute kidney injury; $\mathrm{SCr}$, serum creatinine.

\section{Renal Recovery}

Overall, 9 studies involving 22,617 patients were pooled. As shown in Figure 3, CA-AKI patients tended to show a similar renal recovery to HA-AKI patients (OR 1.27, 95\% CI 0.53$\left.3.02, I^{2}=99 \%, p=0.59\right)$. Exclusion of studies with a small sample size $(n=150)$ did not change the effect size nor the degree of heterogeneity (OR 1.14, 95\% CI 0.46-2.86, $I^{2}=99 \%, p=0.78$ ). The subgroup analysis of different AKI definitions provided inconsistent results. The studies that required $\mathrm{SCr}$ over $2 \mathrm{mg} / \mathrm{dL}$ as the AKI definition showed significantly better renal recovery of CA-AKI compared with HA-AKI patients (OR 4.97, 95\% CI 1.95-12.66, $I^{2}=74 \%$, $p=0.0008$ ). The studies that applied the KDIGO or RIFLE criteria showed no difference in renal recovery between CA- and HA-AKI patients (KDIGO: OR 0.69, 95\% CI 0.22-2.14, $I^{2}=$ $99 \%, p=0.52$; RIFLE: OR $1.22,95 \%$ CI $\left.0.35-4.30, I^{2}=98 \%, p=0.76\right)$. No publication bias was detected based on Egger's test $(p=0.214)$. 


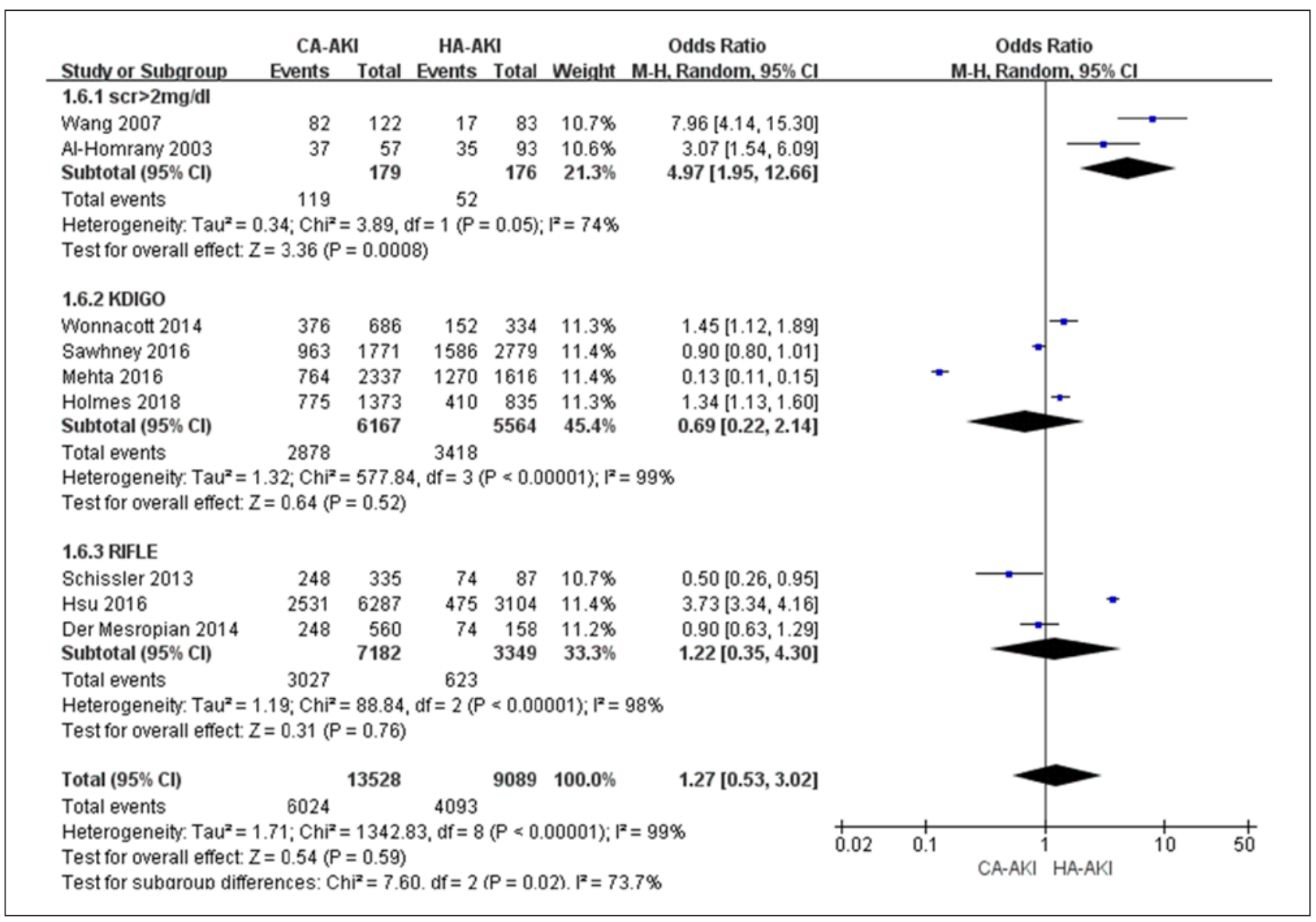

Fig. 3. Forest plot of renal recovery between CA- and HA-AKI patients. HA-AKI, hospital-acquired acute kidney injury; CA-AKI, community-acquired acute kidney injury; SCr, serum creatinine.

Oliguria

Three studies involving 630 patients were pooled for oliguria. In the overall analysis, CA-AKI patients showed a significantly lower rate of oliguria occurrence than HA-AKI patients (OR 0.58, 95\% CI 0.38-0.88, $I^{2}=28 \%, p=0.01$ ). Subgroup analyses of AKI definitions showed that Sesso et al. [31] reported no differences in the oliguria rate between CA- and HA-AKI patients (OR 0.76, 95\% CI 0.49-1.17, $p=0.27$ ). The other 2 studies, which required $\mathrm{SCr}>2$ $\mathrm{mg} / \mathrm{dL}$, showed consistent result with the overall analysis (OR $0.45,95 \% \mathrm{CI} 0.27-0.75, I^{2}=$ $0 \%, p=0.002$ ). Figure 4 illustrates the details. No publication bias was identified based on Egger's test $(p=0.275)$.

Dialysis

Eleven studies involving 28,834 patients were pooled for dialysis. Our analysis indicated that both groups of AKI patients were similarly in the need of dialysis treatment (OR 1.05, $95 \%$ CI $0.82-1.34, I^{2}=60 \%, p=0.70$; Fig. 5). Exclusion of studies with a small sample size $(n=100)$ did not change the effect size or the degree of heterogeneity (OR 1.07, 95\% CI $0.83-$ $1.37, I^{2}=62 \%, p=0.60$ ). In the subgroup analysis of AKI definitions, studies that applied the KDIGO criteria showed a significantly higher need of dialysis for CA-AKI patients, which is exactly the opposite of the result obtained in studies using RIFLE (KDIGO: OR 1.35, 95\% CI $1.16-1.57, I^{2}=0 \%, p<0.0001$; RIFLE: OR $0.67,95 \%$ CI $\left.0.50-0.89, I^{2}=0 \%, p=0.006\right)$. Studies 


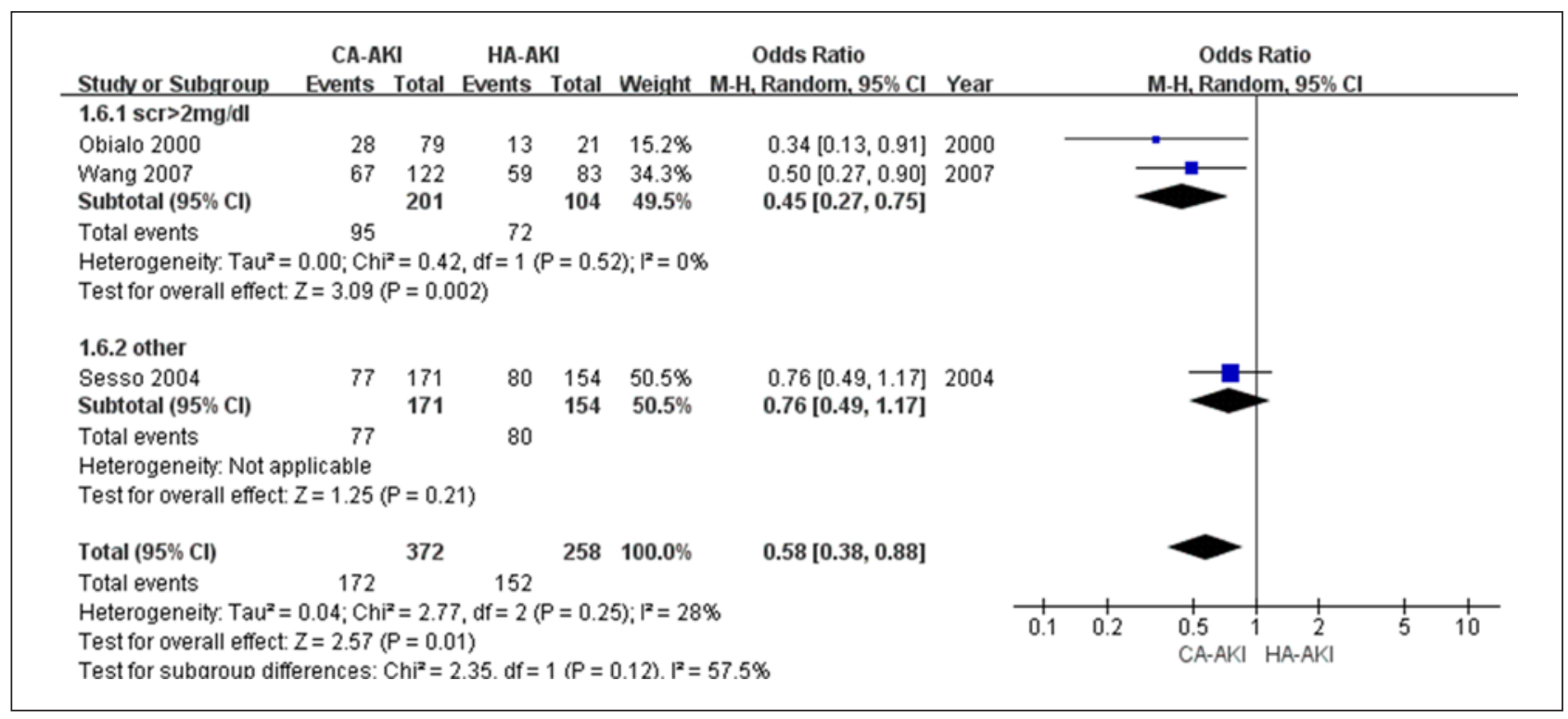

Fig. 4. Forest plot of the oliguria rate between CA- and HA-AKI patients. HA-AKI, hospital-acquired acute kidney injury; CA-AKI, community-acquired acute kidney injury; SCr, serum creatinine.

that required $\mathrm{SCr}>2 \mathrm{mg} / \mathrm{dL}$ and Sesso et al. [31] both showed consistent results with the overall analysis (SCr >2 mg/dL: OR 0.90, 95\% CI 0.61-1.33, $I^{2}=0 \%, p=0.60$; Sesso et al. [31]: OR $1.50,95 \%$ CI $0.97-2.33, p=0.07)$. Egger's test did not show a publication bias $(p=0.381)$.

\section{ICU Risk}

In total, 8 studies involving 22,164 patients were pooled. As shown in Figure 6, ICU need was significantly lower in CA-AKI patients (OR 0.24, 95\% CI 0.14-0.40, $I^{2}=96 \%, p<0.00001$ ). Exclusion of studies with a small sample size did not change the effect size or the degree of heterogeneity (OR 0.27, 95\% CI 0.16-0.47, $I^{2}=96 \%, p<0.00001$ ). Subgroup analysis of different AKI definitions showed consistent results with the overall analysis $(\mathrm{SCr}>2 \mathrm{mg} / \mathrm{dL}$ : OR 0.08, 95\% CI 0.03-0.23, $I^{2}=72 \%, p<0.00001$; KDIGO: OR 0.40, 95\% CI 0.18-0.89, $I^{2}=$ $98 \%, p=0.02$; RIFLE: OR $0.32,95 \%$ CI $\left.0.24-0.44, I^{2}=0 \%, p<0.00001\right)$. The study by Schissler et al. [32] showed that for patients at stage of "Failure" classified by RIFLE criteria, CA-AKI patients were in higher need of ICU (OR 31.85, 95\% CI 1.87-542.24, $p=0.002$ ). In contrast, for patients at the other 2 stages of "Risk" and "Injury", CA-AKI patients were less inclined to require the ICU (RIFLE R: OR 0.28, 95\% CI 0.13-0.62, $p=0.002$; RIFLE I: OR 0.33, 95\% CI $0.15-0.73, p=0.006)$. No publication bias was observed $(p=0.059)$.

\section{Length of Hospital Stay}

Altogether, 9 studies provided data for the length of hospital stay. Figure 7 shows a significantly shorter hospitalization period for CA-AKI patients (MD -9.42, 95\% CI -13.73 to -5.12, $\left.I^{2}=98 \%, p<0.0001\right)$. No publication bias was also observed ( $\left.p=0.077\right)$. Exclusion of studies with a small sample size $(n=100)$ did not change the effect size or the degree of heterogeneity (OR $-9.11,95 \%$ CI -13.56 to $-4.67, I^{2}=98 \%, p<0.001$ ). For the subgroup analysis of different AKI definitions, studies that applied the KDIGO or RIFLE criteria showed consistent results with the overall analysis (KDIGO: MD $-5.27,95 \% \mathrm{CI}-9.15$ to $-1.40, p=0.008, I^{2}=97 \%$; RIFLE: MD $-11.13,95 \%$ CI -15.92 to $\left.-6.34, p<0.00001, I^{2}=87 \%\right)$. Studies that required $\mathrm{SCr}>2 \mathrm{mg} /$ $\mathrm{dL}$ showed no significant difference in length of hospitalization stay between CA- and HA-AKI 


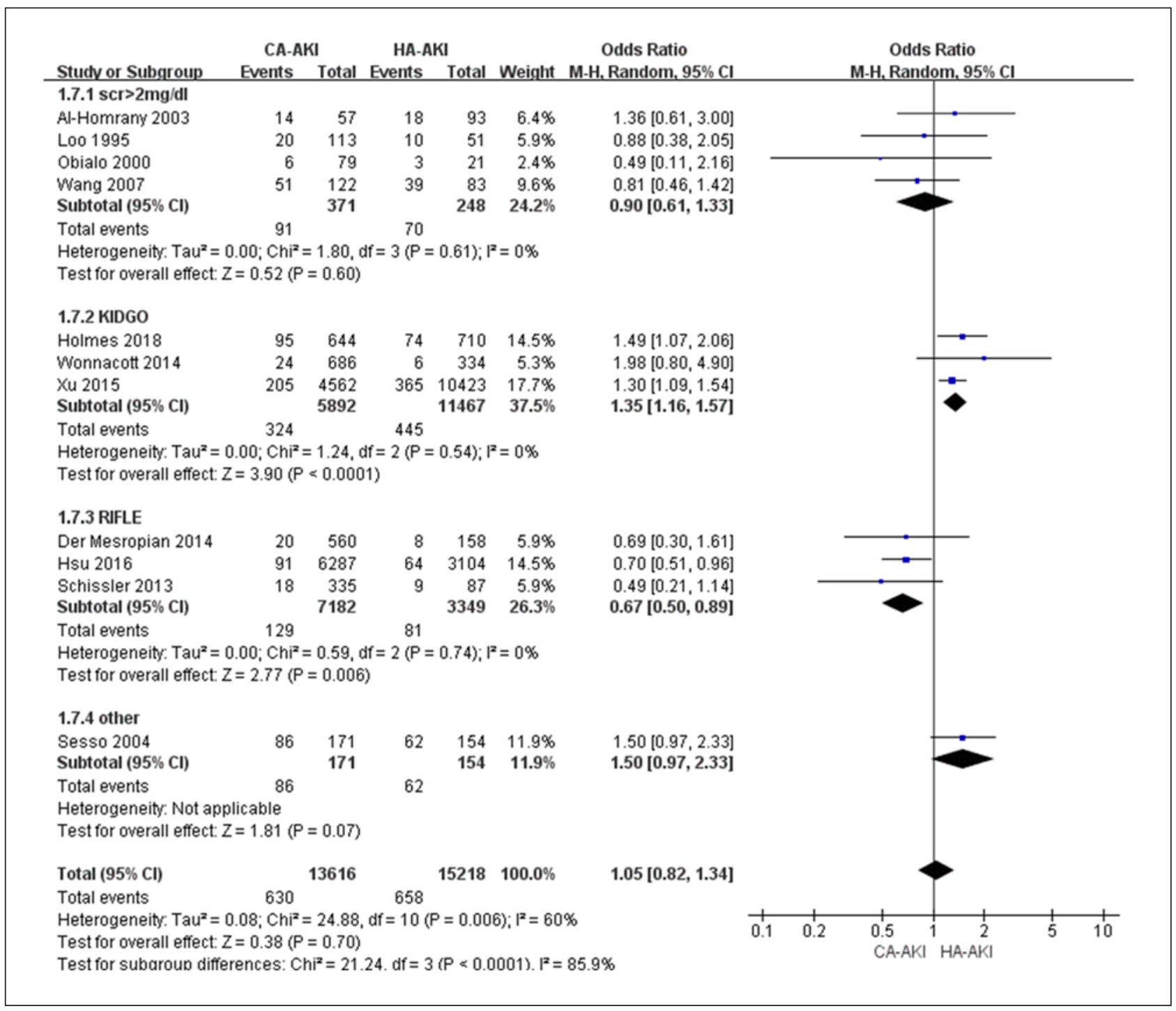

Fig. 5. Forest plot of the need for dialysis between CA- and HA-AKI patients. HA-AKI, hospital-acquired acute kidney injury; CA-AKI, community-acquired acute kidney injury; SCr, serum creatinine.

patients (MD -13.97, 95\% CI -29.16 to $1.22, I^{2}=86 \%, p=0.07$ ). The study by Schissler et al. [32] showed that for patients at stage of "Risk" classified by RIFLE criteria, CA- and HA-AKI patients had a similar length of hospital stay (OR $-1.80,95 \%$ CI -3.94 to $0.34, p=0.10$ ). In contrast, for patients at the other 2 stages of "Injury" and "Failure," CA-AKI patients tended to have a shorter hospital stay than HA-AKI patients (RIFLE I: OR $-7.00,95 \% \mathrm{CI}-12.43$ to $-1.57, p=0.01$; RIFLE F: OR $-18.3095 \%$ CI -30.83 to $-5.77, p=0.004$ ).

\section{Risk of Bias}

Risk of bias ratings for each study were assessed with the Nosgen-ranking tool. All the studies were observational, including ten retrospective cohort, 3 prospective, and 2 crosssectional studies. Considering the similar data content calculated in the 2 cross-sectional studies, they were regarded as cohort studies when assessing bias. The included studies collected statistics from hospitals covering large local populations and equipped with well- 


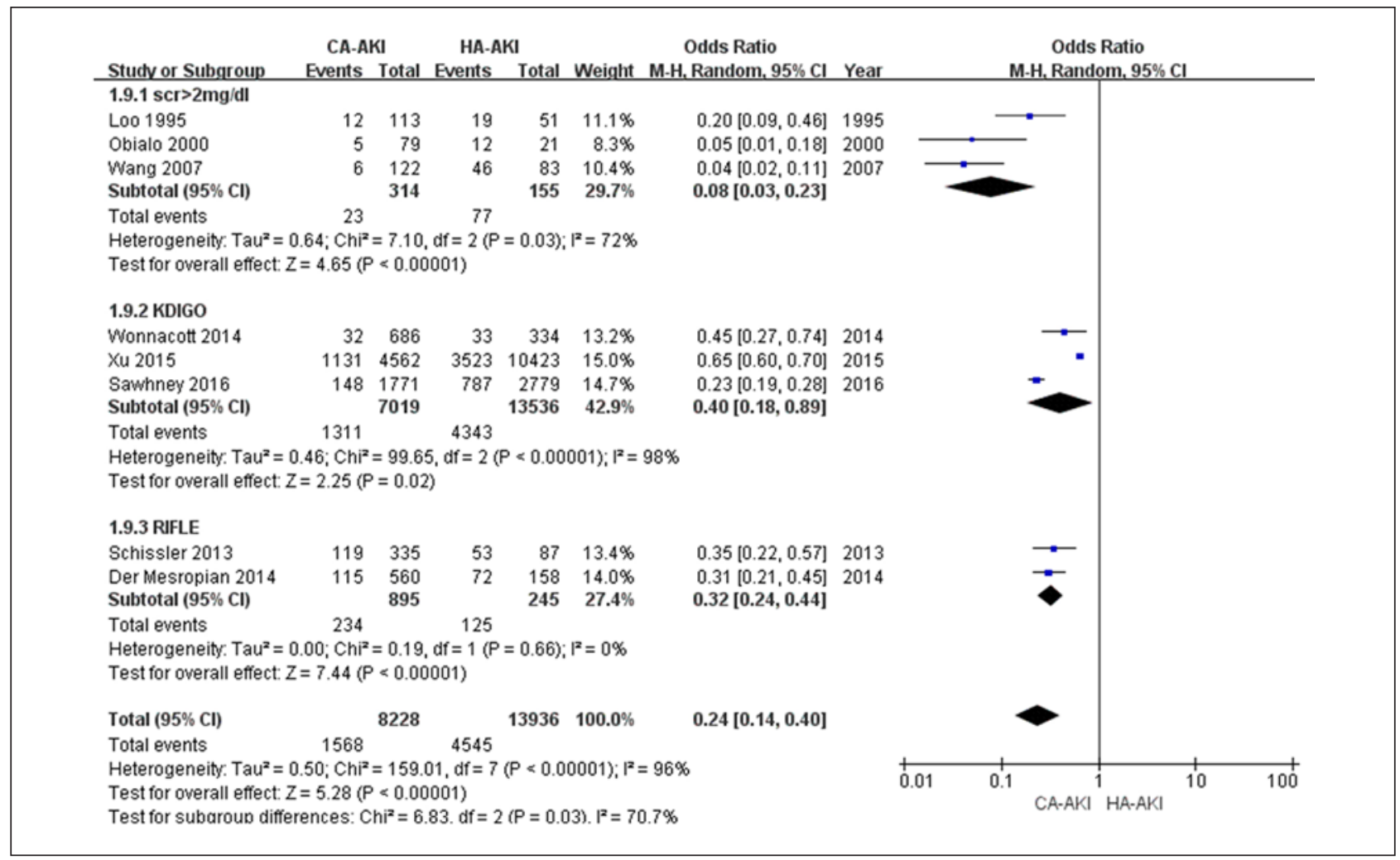

Fig. 6. Forest plot of ICU treatment between CA- and HA-AKI patients. HA-AKI, hospital-acquired acute kidney injury; CA-AKI, community-acquired acute kidney injury; $\mathrm{SCr}$, serum creatinine.

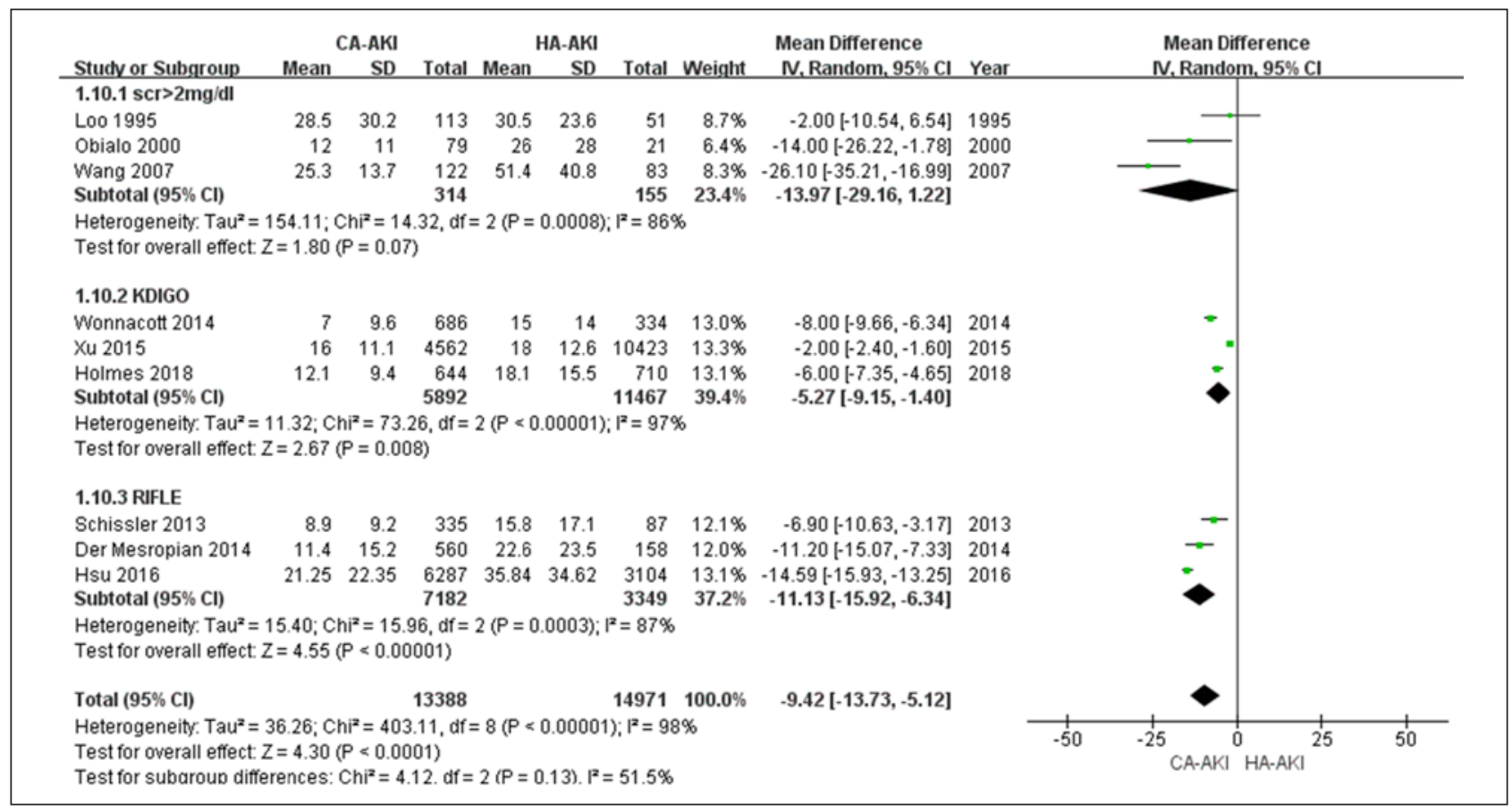

Fig. 7. Forest plot of length of hospital stay between CA- and HA-AKI patients. HA-AKI, hospital-acquired acute kidney injury; CA-AKI, community-acquired acute kidney injury; $\mathrm{SCr}$, serum creatinine. 


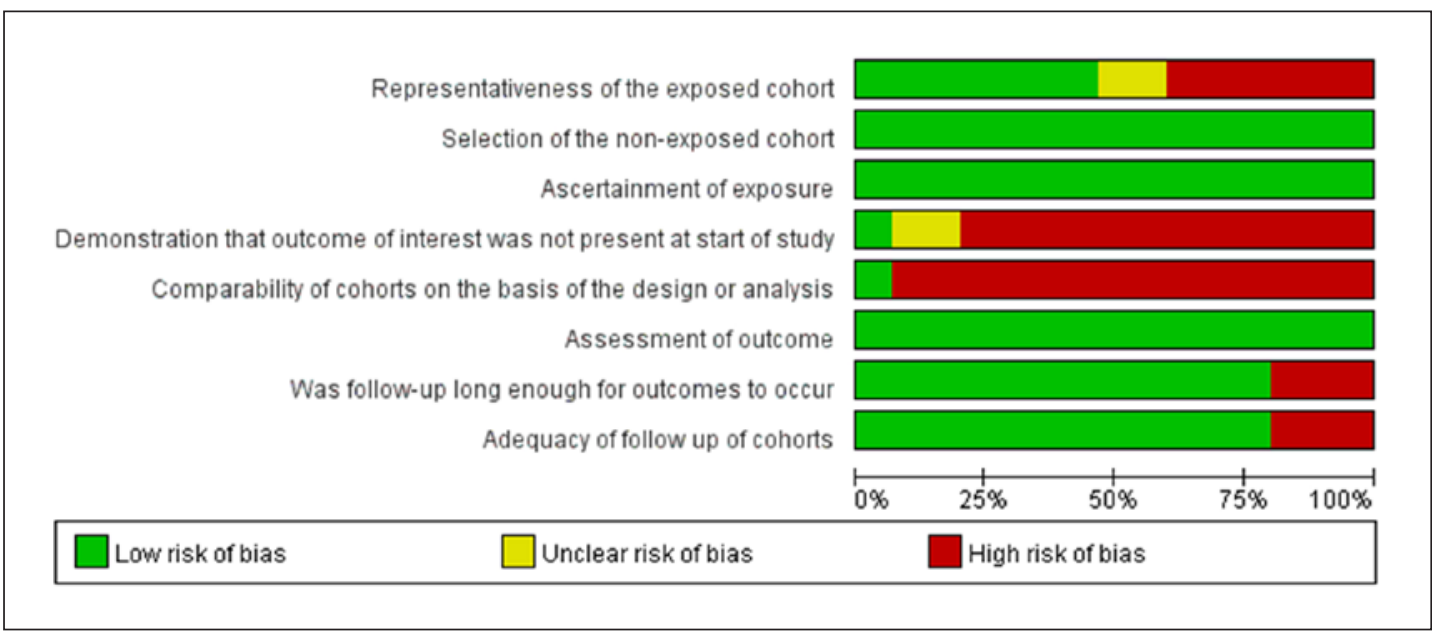

Fig. 8. Risk of bias graph for the included studies.

applied electronic medical systems that documented patients' detailed information. We considered the selection bias to be low risk but the comparability to be high risk. Regarding outcomes, all the included studies provided complete and adequate hospitalization information, and therefore we assessed the outcome bias as low risk. The details are presented in Figures 8 and 9.

\section{Discussion}

With the advances in the research and recognition of AKI, CA-AKI has aroused similar attention and importance as HA-AKI. This meta-analysis provides evidence for the different clinical characteristics and prognosis between CA and HA-AKI patients. In our study, we included 1 study consisting of data collected worldwide [12]; 5 studies conducted in Asia [5, $13,17,18,33]$; another 5 studies in America [19, 20, 25, 31, 32], including 1 specializing in the African-American population [25]; 3 studies in the United Kingdom [27-29]; and 1 study in Saudi Arabia [14]. Overall, our study managed to cover a broad population. For each outcome, the results did not show a significant change after we excluded the relatively smaller samples. Considering the different AKI definitions applied by the included studies, we conducted subgroup analyses to reveal potential disagreements among different AKI definitions in terms of performance. A subgroup analysis for separate stages of AKI was also conducted if data were available.

Our study supported a relatively worse prognosis for HA-AKI patients. The mortality of HA-AKI patients was higher. In the subgroup analysis of various AKI definitions, the results remained in accordance with the overall analysis of mortality. In addition, HA-AKI patients showed more severe clinical manifestations, with a higher oliguria rate, greater demand for the ICU, as well as a longer hospitalized stay. Although in the subgroup analysis of the study by Schissler et al. [32], CA-AKI patients in RIFLE's "Failure" stage needed more ICU admissions than HA-AKI patients in the same stage, this result has a much weaker statistical value, given its wider $95 \% \mathrm{CI}$ and the fact that it stems from only one study. For renal recovery, oliguria, and length of hospital stay, inconsistency was drawn by using different diagnostic criteria. Two studies requiring SCr over $2 \mathrm{mg} / \mathrm{dL}$ showed significantly better renal recovery of CA-AKI compared with HA-AKI patients, while the other studies using the RIFLE or KDIGO 
Kidney

Blood Pressure

Research

\begin{tabular}{l|l}
\hline Kidney Blood Press Res 2019;44:879-896 \\
\hline DOI: 10.1159/000502546 & $\begin{array}{l}\text { @ 2019 The Author(s). Published by S. Karger AG, Basel } \\
\text { www.karger.com/kbr }\end{array}$ \\
\hline
\end{tabular}

Huang et al.: Comparison of CA-AKI and HA-AKI

Fig. 9. Risk of bias summary for

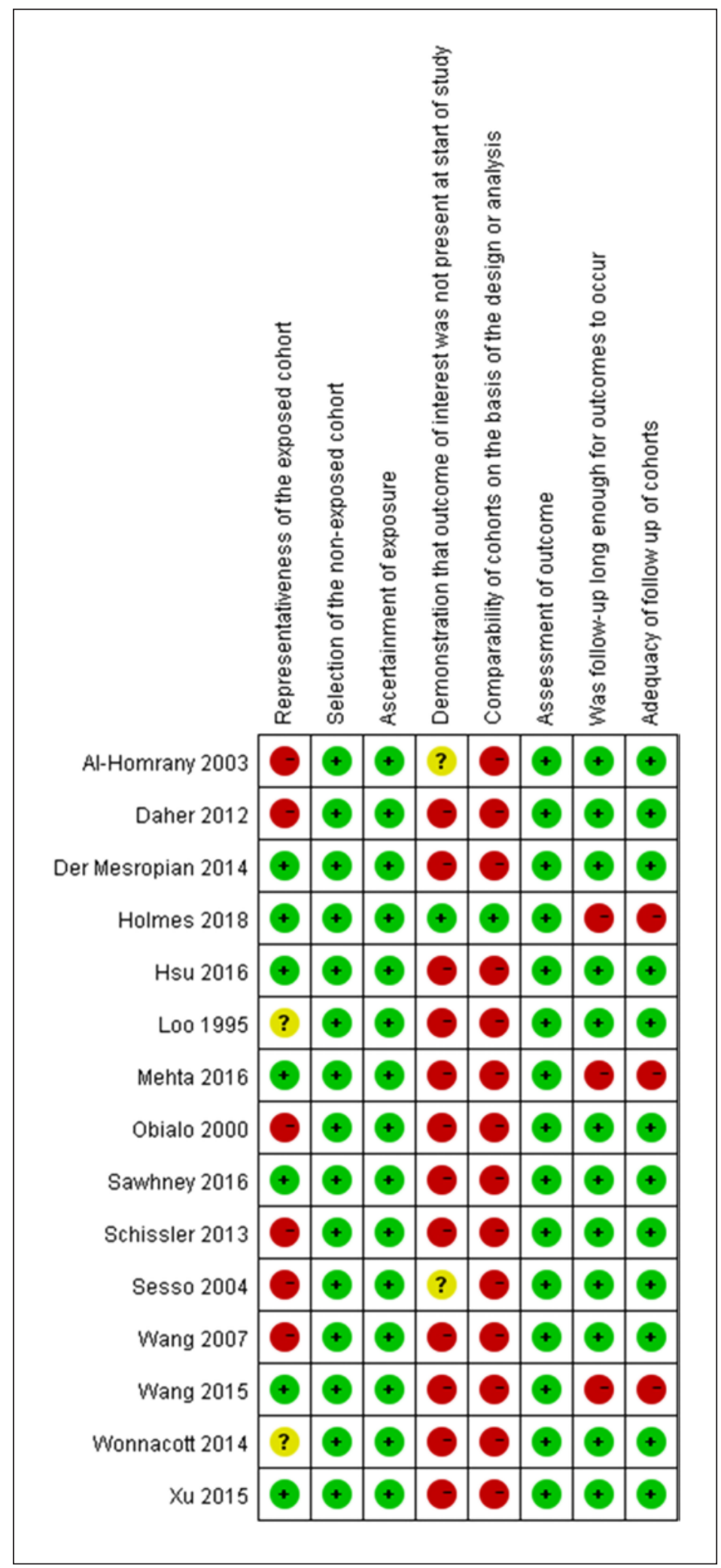




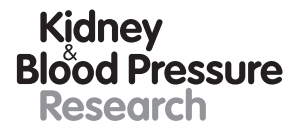

Kidney
Blood Pressure

Research \begin{tabular}{l|l}
\hline Kidney Blood Press Res 2019:44:879-896 \\
\hline DOI: 10.1159/000502546 & $\begin{array}{l}\text { @ 2019 The Author(s). Published by S. Karger AG, Basel } \\
\text { www.karger.com/kbr }\end{array}$ \\
\hline
\end{tabular}

Huang et al.: Comparison of CA-AKI and HA-AKI

criteria showed no difference. Sesso et al. [31] alone reported no difference in oliguria rate between CA- and HA-AKI patients, while the other 2 studies using $\mathrm{SCr}>2 \mathrm{mg} / \mathrm{dL}$ showed a significantly lower rate of oliguria occurrence in CA-AKI. As the recognition of AKI develops, the definition of AKI becomes more specific and sensitive. The subsequent criteria such as the RIFLE or KDIGO perform better in a consensus of identifying AKI occurrence as well as clinical characteristics than the previously used ones.

An exceptional inconsistent result between the RIFLE and KDIGO criteria was presented in dialysis. In studies using the KIDGO criteria, CA-AKI was associated with a higher need for dialysis compared with HA-AKI, but were with a lower need in studies using the RIFLE criteria. It can be attributed to the later publish of the KDIGO criteria [26] than the RIFLE criteria [30]. The therapeutic efficacy of dialysis, especially continuous renal replacement therapy (RRT), was first applied and emphasized in the treatment of HA-AKI patients in ICU [34]. With the accumulation of evidence from numerous clinical researches, dialysis becomes more and more widely used in the rescue of all kinds of AKI patients, including CA-AKI. Different from HA-AKI patients, who may appear in every department in the hospital, most CA-AKI patients are admitted to the nephrology department and treated by nephrologists. These patients are usually better recognized and have more access to dialysis. This might contribute to the higher rate of appliance of dialysis in CA-AKI patients in the KDIGO subgroup than the RIFLE subgroup. However, we noticed that no significant difference in renal recovery was found between KDIGO and RIFLE subgroups. Factors associated with renal recovery and patients prognosis after an AKI episode are complicated, including inherent risk factors (e.g., age, CKD status, or comorbidities such as diabetes), modifiable patients factors (e.g., anemia, sepsis, or number of organ failure), and process of care (e.g., hemodialysis dose and modality, or nephrotoxic drug exposure) [35]. Hemodialysis provides definitive benefits to recovery, but the optimal dialysis dose, modality, and initiation time were still under debate. There was even less evidence in peritoneal dialysis [36, 37]. The RENAL study [38] and the ATN study [39] showed that an improvement in patient survival or renal recovery could not be achieved by increasing the intensity of RRT. Moreover, a recent meta-analysis [40] and a large-scale randomized controlled trial (RCT) [41] revealed no added benefit of early initiation of RRT for patients with AKI. Therefore, despite the inconsistency of dialysis risk between CA-AKI and HA-AKI, dialysis may not sufficiently affect the recovery rate of AKI patients. More well-designed RCTs with sufficient follow-up are necessary to evaluate the recovery of AKI patients between CA- and HA-AKI.

Another systematic review and meta-analysis, conducted by Inokuchi et al. [42], also examined the difference between CA and HA-AKI patients in terms of patient characteristics, in-hospital mortality, and hospital length of stay. Eight eligible observational studies with 17,939 patients were enrolled. We acquired similar results for comparisons of in-hospital mortality and length of hospital stay. Furthermore, our study included a larger number of studies and also covered a larger population, which may provide more convincing advice for the clinicians. Moreover, clinical characteristics were covered in more detail, such as renal recovery, oliguria rate, dialysis need, and ICU risk. We believe these details will help to form a comprehensive understanding of the discrepancies of the 2 types of AKI.

There are several limitations to our study. First, we combined studies with certain degrees of heterogeneity. Retrospective studies encompassed the majority, and 3 prospective observational studies were included $[14,29,31]$. The inconsistency among different definitions of AKI and renal recovery also contributed to the variations of our results. The difference among different hospitals, age and gender distribution, ethnicities screened, and sampling error may all contribute to the high heterogeneity. Thus, more sensitive and precise kidney monitoring may allow better detection. Second, the total sample size was moderate. Third, sampling bias may exist due to the limited studies in this meta-analysis, and more studies are required to verify our results in the future. 


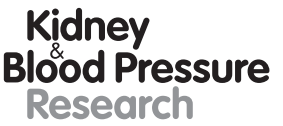

\begin{tabular}{l|l}
\hline Kidney Blood Press Res 2019;44:879-896 \\
\hline DOI: 10.1159/000502546 & $\begin{array}{l}\text { @ 2019 The Author(s). Published by S. Karger AG, Basel } \\
\text { www.karger.com/kbr }\end{array}$ \\
\hline
\end{tabular}

Huang et al.: Comparison of CA-AKI and HA-AKI

\section{Conclusions and Implications for Future Research}

Significant differences exist in the clinical characteristics between CA and HA-AKI. CA-AKI patients showed less severe clinical manifestations and a better prognosis in comparison to HA-AKI patients. Indicators such as mortality, oliguria incidence, ICU need, and length of hospital stay all showed better results for CA-AKI patients. Concerning renal recovery, CA and HA-AKI patients showed no significant difference. The optimal timing and dosage of dialysis to improve prognosis of AKI patients need further evidence and debate. The subgroup analysis of AKI definitions indicated that the KDIGO and RIFLE criteria were nearly consistent in assessing AKI clinical conditions and predicting prognosis. However, the distinct results for dialysis between these 2 might be partly due to the later publish of KDIGO criteria than RIFLE criteria. More studies, particularly multicenter RCTs, are required to illustrate potential differences in the renal recovery of CA- and HA-AKI patients as well as in the performance of diverse AKI definitions.

\section{Statement of Ethics}

The authors have no ethical conflicts to declare.

\section{Disclosure Statement}

The authors declare no conflict of interest.

\section{Funding Sources}

Z.M. is a fellow of the International Society of Nephrology, and this work was funded in part by the National Nature Science Fund of China (Nos. 81570621, 81770670, 81700579, and 81200499) and the Outstanding Young Investigator Fund of the Shanghai Municipal Health Planning Commission (2018YQ25).

\section{Author Contributions}

Z.M.: acts as guarantor for the validity of the study report. Z.M. and J.X.: study concept and design. L.H. and C.X.: acquisition of data. M.R. and J.K.: extraction of data. J.X. and C.X.: checking of data. C.X. and L.H.: analysis and interpretation of data. L.H.: drafting of the manuscript. Z.M. and C.M.: critical revision of the manuscript for important intellectual content.

\section{References}

1 Ali T, Khan I, Simpson W, Prescott G, Townend J, Smith W, et al. Incidence and outcomes in acute kidney injury: a comprehensive population-based study. J Am Soc Nephrol. 2007 Apr;18(4):1292-8.

2 Lo LJ, Go AS, Chertow GM, McCulloch CE, Fan D, Ordoñez JD, et al. Dialysis-requiring acute renal failure increases the risk of progressive chronic kidney disease. Kidney Int. 2009 Oct; 76(8):893-9.

3 Lafrance JP, Miller DR. Defining acute kidney injury in database studies: the effects of varying the baseline kidney function assessment period and considering CKD status. Am J Kidney Dis. 2010 0ct;56(4):651-60.

4 Bellomo R, Kellum JA, Ronco C. Acute kidney injury. Lancet. 2012 Aug;380(9843):756-66.

$5 \mathrm{Xu}$ X, Nie S, Liu Z, Chen C, Xu G, Zha Y, et al. Epidemiology and Clinical Correlates of AKI in Chinese Hospitalized Adults. Clin J Am Soc Nephrol. 2015 Sep;10(9):1510-8. 


\begin{tabular}{l|l}
\hline \multicolumn{2}{l}{ Kidney Blood Press Res 2019;44:879-896 } \\
\hline DOI: 10.1159/000502546 & $\begin{array}{l}\text { (c) 2019 The Author(s). Published by S. Karger AG, Basel } \\
\text { www.karger.com/kbr }\end{array}$ \\
\hline
\end{tabular}

Huang et al.: Comparison of CA-AKI and HA-AKI

6 Nolan CR, Anderson RJ. Hospital-acquired acute renal failure. J Am Soc Nephrol. 1998 Apr;9(4):710-8.

7 Liaño F, Pascual J; Madrid Acute Renal Failure Study Group. Epidemiology of acute renal failure: a prospective, multicenter, community-based study. Kidney Int. 1996 Sep;50(3):811-8.

8 Mehta RL, Cerdá J, Burdmann EA, Tonelli M, García-García G, Jha V, et al. International Society of Nephrology's 0by25 initiative for acute kidney injury (zero preventable deaths by 2025): a human rights case for nephrology. Lancet. 2015 Jun;385(9987):2616-43.

9 Hou SH, Bushinsky DA, Wish JB, Cohen JJ, Harrington JT. Hospital-acquired renal insufficiency: a prospective study. Am J Med. 1983 Feb;74(2):243-8.

10 Kaufman J, Dhakal M, Patel B, Hamburger R. Community-acquired acute renal failure. Am J Kidney Dis. 1991 Feb;17(2):191-8.

11 Feest TG, Round A, Hamad S. Incidence of severe acute renal failure in adults: results of a community based study. BMJ. 1993 Feb;306(6876):481-3.

12 Mehta RL, Burdmann EA, Cerdá J, Feehally J, Finkelstein F, García-García G, et al. Recognition and management of acute kidney injury in the International Society of Nephrology 0by25 Global Snapshot: a multinational cross-sectional study. Lancet. 2016 May;387(10032):2017-25.

13 Loo CS, Zainal D. Acute renal failure in a teaching hospital. Singapore Med J. 1995 Jun;36(3):278-81.

14 Al-Homrany M. Epidemiology of acute renal failure in hospitalized patients: experience from southern Saudi Arabia. East Mediterr Health J. 2003 Sep-Nov;9(5-6):1061-7.

15 Jha V, Malhotra HS, Sakhuja V, Chugh KS. Spectrum of hospital-acquired acute renal failure in the developing countries—chandigarh study. Q J Med. 1992 Jul;83(303):497-505.

16 Susantitaphong P, Cruz DN, Cerda J, Abulfaraj M, Alqahtani F, Koulouridis I, et al.; Acute Kidney Injury Advisory Group of the American Society of Nephrology. World incidence of AKI: a meta-analysis. Clin J Am Soc Nephrol. 2013 Sep;8(9):1482-93.

17 Hsu CN, Lee CT, Su CH, Wang YC, Chen HL, Chuang JH, et al. Incidence, Outcomes, and Risk Factors of CommunityAcquired and Hospital-Acquired Acute Kidney Injury: A Retrospective Cohort Study. Medicine (Baltimore). 2016 May;95(19):e3674.

18 Yang L, Xing G, Wang L, Wu Y, Li S, Xu G, et al.; ISN AKF 0by25 China Consortiums. Acute kidney injury in China: a cross-sectional survey. Lancet. 2015 Oct;386(10002):1465-71.

19 Daher EF, Silva Junior GB, Santos SQ, R Bezerra CC, Diniz EJ, Lima RS, et al. Differences in community, hospital and intensive care unit-acquired acute kidney injury: observational study in a nephrology service of a developing country. Clin Nephrol. 2012 Dec;78(6):449-55.

20 Der Mesropian PJ, Kalamaras JS, Eisele G, Phelps KR, Asif A, Mathew RO. Long-term outcomes of communityacquired versus hospital-acquired acute kidney injury: a retrospective analysis. Clin Nephrol. 2014 Mar; 81(3):174-84.

21 Kellum JA, Lameire N; KDIGO AKI Guideline Work Group. Diagnosis, evaluation, and management of acute kidney injury: a KDIGO summary (Part 1). Crit Care. 2013 Feb;17(1):204.

22 Mehta RL, Kellum JA, Shah SV, Molitoris BA, Ronco C, Warnock DG, et al.; Acute Kidney Injury Network. Acute Kidney Injury Network: report of an initiative to improve outcomes in acute kidney injury. Crit Care. 2007; 11(2):R31.

23 Wang Y, Cui Z, Fan M. Retrospective analysis on Chinese patients diagnosed with acute renal failure hospitalized during the last decade (1994-2003). Am J Nephrol. 2005 Sep-Oct;25(5):514-9.

24 Wang Y, Cui Z, Fan M. Hospital-acquired and community-acquired acute renal failure in hospitalized Chinese: a ten-year review. Ren Fail. 2007;29(2):163-8.

25 Obialo CI, Okonofua EC, Tayade AS, Riley LJ. Epidemiology of de novo acute renal failure in hospitalized African Americans: comparing community-acquired vs hospital-acquired disease. Arch Intern Med. 2000 May; 160(9): 1309-13.

26 Kidney Disease: Improving Global Outcomes (KDIGO) Acute Kidney Injury Work Group (2012) KDIGO clinical practice guideline for acute kidney injury. Available from: http://www.kidney-international.org.

27 Sawhney S, Fluck N, Fraser SD, Marks A, Prescott GJ, Roderick PJ, et al. KDIGO-based acute kidney injury criteria operate differently in hospitals and the community-findings from a large population cohort. Nephrol Dial Transplant. 2016 Jun;31(6):922-9.

28 Wonnacott A, Meran S, Amphlett B, Talabani B, Phillips A. Epidemiology and outcomes in community-acquired versus hospital-acquired AKI. Clin J Am Soc Nephrol. 2014 Jun;9(6):1007-14.

29 Holmes J, Roberts G, Geen J, Dodd A, Selby NM, Lewington A, et al.; Welsh AKI steering group. Utility of electronic AKI alerts in intensive care: A national multicentre cohort study. J Crit Care. 2018 Apr;44:185-90.

30 Bellomo R, Ronco C, Kellum JA, Mehta RL, Palevsky P; Acute Dialysis Quality Initiative workgroup. Acute renal failure - definition, outcome measures, animal models, fluid therapy and information technology needs: the Second International Consensus Conference of the Acute Dialysis Quality Initiative (ADQI) Group. Crit Care. 2004 Aug;8(4):R204-12.

31 Sesso R, Roque A, Vicioso B, Stella S. Prognosis of ARF in hospitalized elderly patients. Am J Kidney Dis. 2004 Sep;44(3):410-9.

32 Schissler MM, Zaidi S, Kumar H, Deo D, Brier ME, McLeish KR. Characteristics and outcomes in communityacquired versus hospital-acquired acute kidney injury. Nephrology (Carlton). 2013 Mar;18(3):183-7.

33 Cui Z, Wang Y, Fan MH. [Comparison of clinical characteristics between hospital-acquired and communityacquired acute renal failure]. Zhongguo Wei Zhong Bing Ji Jiu Yi Xue. 2005 Oct;17(10):615-8. 
34 Ronco C, Bellomo R, Homel P, Brendolan A, Dan M, Piccinni P, et al. Effects of different doses in continuous veno-venous haemofiltration on outcomes of acute renal failure: a prospective randomised trial. Lancet. 2000 Jul;356(9223):26-30.

35 Macedo E, Mehta RL. Renal Recovery after Acute Kidney Injury. Contrib Nephrol. 2016;187:24-35.

36 Liu L, Zhang L, Liu GJ, Fu P. Peritoneal dialysis for acute kidney injury. Cochrane Database Syst Rev. 2017 Dec; 12:CD011457.

37 Xue C, Gu YY, Cui CJ, Zhou CC, Wang XD, Ruan MN, et al. New-onset glucose disorders in peritoneal dialysis patients: a meta-analysis and systematic review. Nephrol Dial Transplant. 2019 Jun;gfz116.

38 Bellomo R, Cass A, Cole L, Finfer S, Gallagher M, Lo S, et al.; RENAL Replacement Therapy Study Investigators. Intensity of continuous renal-replacement therapy in critically ill patients. N Engl J Med. 2009 Oct;361(17): 1627-38.

39 Palevsky PM, Zhang JH, O’Connor TZ, Chertow GM, Crowley ST, Choudhury D, et al.; VA/NIH Acute Renal Failure Trial Network. Intensity of renal support in critically ill patients with acute kidney injury. N Engl J Med. 2008 Jul;359(1):7-20.

40 Bhatt GC, Das RR. Early versus late initiation of renal replacement therapy in patients with acute kidney injury-a systematic review \& meta-analysis of randomized controlled trials. BMC Nephrol. 2017 Feb;18(1):78.

41 Barbar SD, Clere-Jehl R, Bourredjem A, Hernu R, Montini F, Bruyère R, et al.; IDEAL-ICU Trial Investigators and the CRICS TRIGGERSEP Network. Timing of Renal-Replacement Therapy in Patients with Acute Kidney Injury and Sepsis. N Engl J Med. 2018 Oct;379(15):1431-42.

42 Inokuchi R, Hara Y, Yasuda H, Itami N, Terada Y, Doi K. Differences in characteristics and outcomes between community- and hospital-acquired acute kidney injury: A systematic review and meta-analysis. Clin Nephrol. 2017 Oct;88(10):167-82. 